\title{
Metabolic Imaging of Infection
}

\author{
Ismaheel Lawal ${ }^{1}$, JanRijn Zeevaart ${ }^{1,2}$, Thomas Ebenhan ${ }^{1}$, Alfred Ankrah ${ }^{1,3}$, Mariza Vorster $^{1}$, Hendrik G. Kruger ${ }^{4}$, \\ Thavendran Govender ${ }^{4}$, and Mike Sathekge ${ }^{1}$ \\ ${ }^{1}$ Department of Nuclear Medicine, University of Pretoria, Pretoria, South Africa; ${ }^{2}$ Radiochemistry, South African Nuclear Energy \\ Corporation SOC Ltd. (Necsa), Pelindaba, Pretoria, South Africa; ${ }^{3}$ Department of Nuclear Medicine and Molecular Imaging, \\ University Medical Center Groningen, University of Groningen, The Netherlands; and ${ }^{4}$ Catalysis and Peptide Research Unit, School \\ of Health Sciences and School of Chemistry and Physics, University of KwaZulu-Natal, Durban, South Africa
}

\begin{abstract}
Metabolic imaging has come to occupy a prominent place in the diagnosis and management of microbial infection. Molecular probes available for infection imaging have undergone a rapid evolution starting with nonspecific agents that accumulate similarly in infection, sterile inflammation, and neoplastic tissue and then extending to more targeted probes that seek to identify specific microbial species. This focus review describes the metabolic and molecular imaging techniques currently available for clinical use in infection imaging and those that have demonstrated promising results in preclinical studies with the potential for clinical applications.
\end{abstract}

Key Words: molecular imaging; infection; anti-microbial; optical imaging

J Nucl Med 2017; 58:1727-1732

DOI: 10.2967/jnumed.117.191635

$\mathbf{T}$ he diagnosis and treatment of infection remain challenging in clinical practice. The gold standard for diagnosis is culture of a specimen obtained from the infection site. Obtaining a sample is often difficult because the site of infection may be unknown or biopsy may be too invasive. Biopsy for microbiologic evaluation may also be fraught with sampling errors leading to false-negative results. Imaging is therefore often necessary in view of these challenges.

Anatomic imaging with radiologic techniques evaluates structural changes in tissue architecture in the assessment of infection. These anatomic changes become apparent only when the infectious process is advanced and tissue damage has occurred. Similarly, restoration of tissue architecture lags behind successful treatment of infection, making anatomic imaging less ideal for early diagnosis and therapy response assessment.

Received Jul. 5, 2017; revision accepted Aug. 14, 2017.

For correspondence or reprints contact: Mike Sathekge, Department of Nuclear Medicine, University of Pretoria and Steve Biko Academic Hospital,

Private Bag X169, Pretoria, 0001, South Africa.

E-mail: mike.sathekge@up.ac.za

Published online Aug. 17, 2017.

COPYRIGHT (c) 2017 by the Society of Nuclear Medicine and Molecular Imaging.
Molecular imaging of infection has been the focus of many human studies, resulting in the introduction of a wide variety of molecular probes in the clinical or preclinical setting for imaging infection.

\section{INFECTION IMAGING: THE INFLAMMATORY CASCADE}

The presence of an infectious organism in the tissue elicits a series of vascular and cellular responses termed inflammation. In response to the presence of microbes, the regional microcirculation undergoes a series of changes aimed at delivering elements of the immune system to the infection site. The vascular events include vasodilation and increased vascular permeability to allow leukocytes and acute-phase proteins to access the infection site. In the cellular events, leukocytes are attracted to the site of infection in large numbers in response to endogenous and exogenous (bacterial products) chemoattractants. Leukocytes are activated at the site of infection, and they increase the oxygen and energy substrate use.

The different steps of this inflammatory cascade have been explored in the development of molecular probes for metabolic imaging of infection. The objectives of molecular imaging of infection include noninvasive and early diagnosis of infection, differentiation of infection from sterile inflammation, monitoring of response to antimicrobial therapy, prognostication, and identification of the offending agent without the need for the often difficult, time-consuming, and sometimes unsuccessful process required to obtain samples and culture the microbe (1).

\section{IMAGING LEUKOCYTE MIGRATION: RADIOLABELED WHITE CELLS}

SPECT imaging of leukocytes radiolabeled with ${ }^{111} \mathrm{In}-$ oxime or ${ }^{99 \mathrm{~m}} \mathrm{Tc}$-hexamethylpropyleneamine oxime is a useful molecular imaging modality in clinical practice.

Radiolabeled leukocyte imaging is the modality of choice in imaging skeletal infection. Triple-phase bone scintigraphy provides sufficient sensitivity and specificity for diagnosing infection in unviolated bones. The specificity of bone scans drops significantly in violated bones such as after trauma or surgery. Radiolabeled leukocyte imaging 
either alone or in combination with marrow imaging provides high sensitivity and specificity above $90 \%$ for diagnosing skeletal infection in this setting $(2,3)$.

Infection of cardiovascular implantable devices is rare. Its occurrence is, however, associated with high morbidity and mortality (4). Radiolabeled leukocyte SPECT/CT provides a highly specific molecular imaging modality for diagnosing infection in this critical condition (5). The success with molecular imaging techniques of cardiac devices has led to the inclusion of positive findings on these modalities as a minor criterion in the modified Duke Criteria for the diagnosis of endocarditis by the European Cardiac Society (6).

The relatively limited resolution of SPECT imaging compared with PET imaging has led to the attempt at labeling white cells with ${ }^{18}$ F-FDG for PET imaging. This endeavor has faced challenges. The labeling efficiency is variable and is lower than what has been described for radiolabeled leukocytes with ${ }^{99 \mathrm{~m} T c-h e x a m e t h y l p r o p y l e n e a m i n e ~ o x i m e ~}$ or ${ }^{111}$ In-oxime (7). Furthermore, ${ }^{18}$ F-FDG elutes from the leukocyte, and the limited half-life of the radionuclide ( $\sim 110 \mathrm{~min}$ ) makes delayed imaging impossible. Concerns also exist about exposure to radiation in the cells being labeled, especially the long-lived lymphocytes $(8)$.

Limitations of radiolabeled leukocytes include a variable uptake in spondylodiskitis; hence, the technique is not used in the evaluation of spinal infection. There is also early diffuse accumulation of the radiolabeled leukocyte in the lungs after reinjection, limiting its application in lung infection imaging. A normal level of circulating leukocytes is necessary for optimum cell labeling. Therefore, radiolabeled leukocyte imaging has a limited role in the evaluation of patients with febrile neutropenia. Moreover, in vitro labeling of leukocytes is laborious and time-consuming and exposes staff to blood-borne infections. These challenges have stimulated interest in the in vivo labeling of leukocytes. Sulesomab is a murine monoclonal IgG antibody fragment that binds to non-cross-reacting antigen 90 on polymorphs and their precursors. ${ }^{99 \mathrm{~m}}$ Tc-labeled sulesomab (LeukoScan; Immunomedics), being an antibody fragment, is able to promptly penetrate into the infectious foci for in vivo labeling of polymorphs.

\section{IMAGING CELLULAR METABOLISM: ${ }^{18} F-F D G$}

The better resolution of PET makes it a more sensitive technique than SPECT in infection imaging. ${ }^{18} \mathrm{~F}-\mathrm{FDG}$ accumulates at sites of infection, sterile inflammation, and neoplastic tissue, resulting in a relatively reduced specificity of ${ }^{18} \mathrm{~F}$-FDG PET as an infection imaging technique. Although radiolabeled leukocyte scintigraphy offers higher specificity, ${ }^{18}$ F-FDG PET imaging provides better sensitivity in the setting of infection imaging (5). The addition of morphologic imaging to either technique in the form of SPECT/CT or PET/CT or, more recently, MRI as PET/ MRI improves the diagnostic accuracy.
${ }^{18} \mathrm{~F}-\mathrm{FDG}$ PET/CT imaging of infection is completed within a relatively short time, is easy to perform, requires no handling of blood, and allows for easy quantification of uptake, which is useful for treatment response assessment. Studies have shown that ${ }^{18} \mathrm{~F}-\mathrm{FDG}$ PET/CT has clinical utility in the evaluation of osteomyelitis. In a head-to-head comparison of ${ }^{18} \mathrm{~F}-\mathrm{FDG}$ PET/CT and MRI in patients with suspected spondylodiskitis, ${ }^{18} \mathrm{~F}-\mathrm{FDG}$ PET/CT outperformed MRI, with a sensitivity and specificity of $96 \%$ and $95 \%$, respectively, for ${ }^{18} \mathrm{~F}$-FDG PET/CT versus $67 \%$ and $84 \%$, respectively, for MRI (9). In a setting of pedal osteomyelitis complicating diabetic foot syndrome, ${ }^{18} \mathrm{~F}-\mathrm{FDG}$ PET/CT has a sensitivity, specificity, and accuracy of $88.3 \%, 96.8 \%$, and $93.8 \%$, respectively (10). In that study, elevated blood sugar levels did not affect the diagnostic performance of the test.

The number of cardiac devices implanted across the world is increasing, and so is infection in these devices. Cardiac device-associated infection requires urgent diagnosis and management. It is also important to differentiate superficial wound infection from an actual device infection, as the two types of infection are managed differently. Whereas deep infection affecting the device requires removal of the device, with its attendant risk of mortality and morbidity, superficial wound infection requires only local wound care and parenteral antibiotics (11). Clinical assessment is often not sufficient to make this distinction (12). In a group of 70 patients with an inconclusive diagnosis of infective endocarditis using the Duke criteria, a study applying ${ }^{18}$ F-FDG PET/CT to these patients was able to reclassify 18 as having infective endocarditis and 45 as having no infective endocarditis (13).

${ }^{18}$ F-FDG PET/CT is highly useful in the evaluation of suspected vascular graft infection. It is also essential in differentiating a superficial wound infection from a deep infection involving the vascular graft. In a prospective study of 32 patients with suspected vascular graft infection, 12 of whom were already on antimicrobial treatment, ${ }^{18} \mathrm{~F}-\mathrm{FDG}$ PET/CT correctly identified 27 patients with infection and ruled out infection in 6 patients (14). Overall, the diagnostic performance of ${ }^{18} \mathrm{~F}-\mathrm{FDG}$ PET/CT in that study using a 5-point visual grading system was as follows: sensitivity of $100 \%$, specificity of $86 \%$, positive predictive value of $96 \%$, negative predictive value of $100 \%$, and accuracy of $97 \%$. Imaging before commencing antimicrobial treatment improved the diagnostic sensitivity. However, this was not confirmed in a more recent study with a larger patient population (15). It is important to know that noninfected prosthetic vascular grafts also demonstrate ${ }^{18}$ F-FDG uptake. A comprehensive description of this physiologic uptake has been presented in a study by Keidar et al. (16).

Fever of unknown origin (FUO) is commonly a result of infection, inflammatory noninfectious conditions, or malignancy. ${ }^{18} \mathrm{~F}-\mathrm{FDG}$ accumulates in all of these conditions, making ${ }^{18} \mathrm{~F}$-FDG PET/CT suitable for the evaluation of patients with FUO. In a recent study of 76 patients with 
FUO, ${ }^{18}$ F-FDG PET/CT led to the final diagnosis of infection as the cause of FUO in $21 \%$ of patients, malignancy in $22 \%$ of patients, inflammatory noninfectious diseases in $12 \%$ of patients, and miscellaneous diagnoses in $5 \%$ of patients (17). A $56 \%$ diagnostic yield was found for ${ }^{18} \mathrm{~F}$ FDG PET/CT in the evaluation of FUO in a metaanalysis of 18 eligible studies including 905 patients (18).

Tuberculosis is an infectious disease that has plagued the human population for many millennia. Despite extensive research into this disease, significant challenges still exist in its diagnosis, treatment, and therapy response assessment (19). The diagnostic gold standard for tuberculosis is culture of the tubercle bacilli, an endeavor that is difficult to achieve because of the difficulty of obtaining the bacilli in a sputum sample or other body fluid or tissue from an infected site. In endemic regions, the diagnosis often relies on suggestive clinical findings and imaging. ${ }^{18}$ F-FDG PET/ CT complements the low yield of bacteriologic confirmation in the initial diagnosis of tuberculosis and follow-up (Fig. 1). In a report of 35 patients evaluated for tuberculosis, ${ }^{18} \mathrm{~F}$-FDG PET/CT was true-positive in 34 patients (20). Both the PET and the CT components of this study were false-negative in one patient with microbiologically confirmed tuberculosis. Overall, PET performed better than $\mathrm{CT}$ in identifying disease in 34 patients, as opposed to

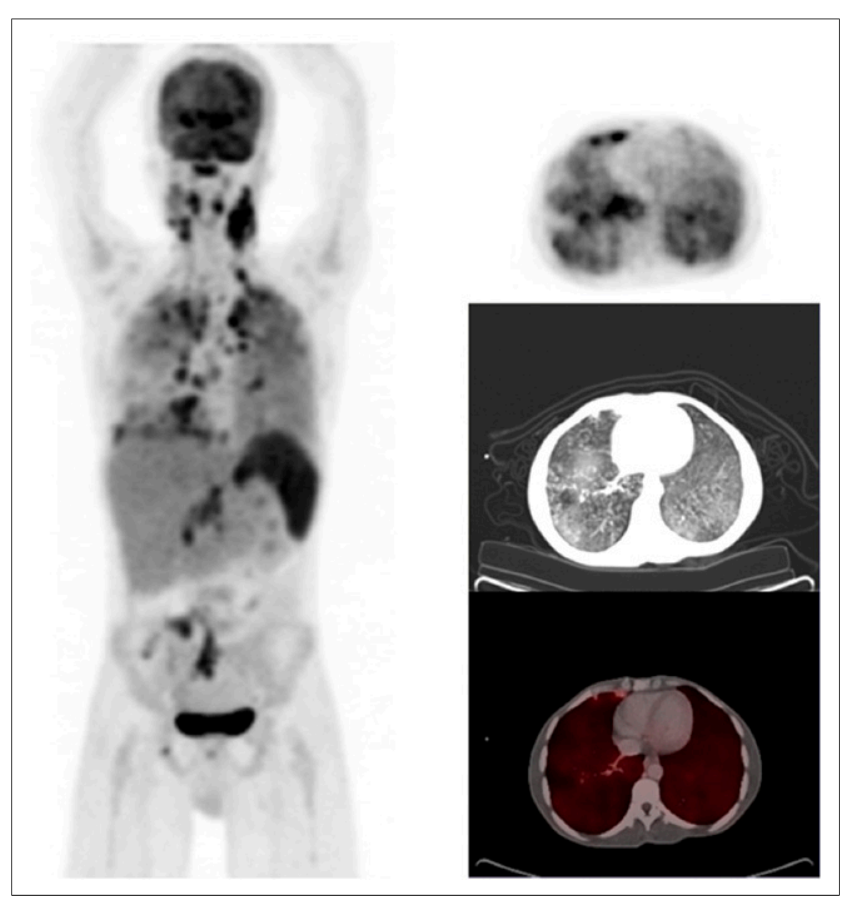

FIGURE 1. 27-y-old HIV-positive patient with suspected recurrence of tuberculosis $3 \mathrm{y}$ after therapy. Maximum-intensityprojection PET and axial fused PET/CT demonstrate diffuse ${ }^{18} \mathrm{~F}-\mathrm{FDG}$-avid lymphadenopathy in cervical, hilar, mediastinal, and abdominal nodes, with intense pulmonary parenchymal splenic uptake. ${ }^{18} \mathrm{~F}-\mathrm{FDG}$ PET/CT demonstrated that tuberculosis was active and upstaged patient by showing extrapulmonary involvement. the 23 identified by CT. Both modalities performed equally in identifying pulmonary tuberculosis, whereas PET performed better in identifying sites of extrapulmonary disease. Identification of sites of extrapulmonary tuberculosis involvement is crucial to management because most sites of extrapulmonary disease require a longer duration of antituberculous therapy.

The tuberculosis granulomatous lesions are highly heterogeneous and undergo changes as they mature (21). They behave differently in response to treatment in the same patient. Although some lesions may respond to therapy, other lesions may progress on treatment. ${ }^{18} \mathrm{~F}$-FDG PET has been shown to be a useful modality in therapy response assessment and outperforms diagnostic CT in this setting (20). In a study of 18 patients with tuberculous lymphadenitis, ${ }^{18}$ F-FDG PET/ CT correctly identified cure in 9 patients (no ${ }^{18} \mathrm{~F}-\mathrm{FDG}$ uptake on posttreatment PET scans) and treatment failure in 2 patients (22). A recent study showed clinical and microbiologic methods to be unreliable in the assessment of cure after successful completion of antituberculous therapy (23). In that study, the investigators performed end-of-treatment ${ }^{18} \mathrm{~F}-\mathrm{FDG}$ PET/CT on patients considered cured on the basis of microbiology assessment and found residual or persistent ${ }^{18} \mathrm{~F}-\mathrm{FDG}$ uptake in lesions. In these patients, persistence of microbial replication was shown by demonstrating Mycobacterium tuberculosis messenger RNA and DNA in respiratory samples obtained by bronchoalveolar lavage.

The presence of HIV infection introduces different dynamics into tuberculosis diagnosis and management. HIV-associated immunosuppression is a common cause of reactivation of latent infection. Patients with latent tuberculosis have isoniazid for chemoprophylaxis. It is, however, important to identify those patients whose disease is subclinical and may progress to clinical tuberculosis after HIV infection. A study evaluated the role of ${ }^{18} \mathrm{~F}$-FDG PET/CT in $35 \mathrm{HIV}$-positive, antiretroviral therapy-naïve patients with latent tuberculosis and identified 10 patients with pulmonary findings suggestive of subclinical tuberculosis (24). There were 2 findings suggestive of subclinical tuberculosis. One was pulmonary infiltrates or fibrotic scars suggestive of bronchogenic reactivation of tuberculosis, and the other was active nodules suggestive of hematogenous spread of tuberculosis. Four of 10 patients with these findings required a full course of antituberculous therapy within 6 mo of follow-up.

\section{IMAGING ANTIBIOTIC BINDING TO MICROBES: RADIOLABELED ANTIMICROBIAL AGENTS}

Antimicrobial peptides have been successfully radiolabeled and tested for infection imaging in animal models and humans. The first radiolabeled antimicrobial agent evaluated for human use was ${ }^{99 \mathrm{~m}} \mathrm{Tc}$-ciprofloxacin. Disappointing results from its application in humans led to its withdrawal from the market. Many other antibiotics, including fluoroquinolones, cephalosporins, and antituberculous drugs, have since been successfully labeled with a suitable radionuclide and tested in preclinical studies $(25,26)$. 


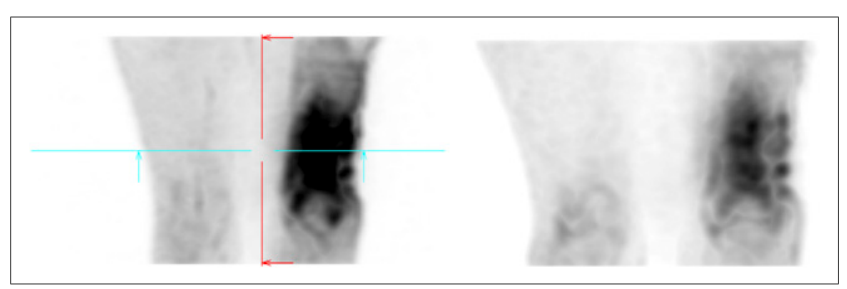

FIGURE 2. $\quad 99 \mathrm{~m} T \mathrm{c}-$-ubiquicidin 29-41 scintigraphy demonstrating intense uptake in left distal femur that corresponded to confirmed osteomyelitis by culture. Significantly reduced uptake is noted on second scan ( 3 wk apart), consistent with partial response and improvement on clinical assessment. ${ }^{99 m}$ Tc-ubiquicidin 29-41 may assume an important role for this indication in osteomyelitis management. (Image provided courtesy of Dr. Enrique Estrada Lobato, IAEA.)

A radiolabeled antimicrobial peptide that has gained popularity in the clinic is radiolabeled ubiquicidin (Fig. 2). Ubiquicidin is a human antimicrobial peptide present in the respiratory epithelium. Its fragments have been successfully labeled with ${ }^{68} \mathrm{Ga}$ for PET imaging and ${ }^{99 \mathrm{~m}} \mathrm{Tc}$ for SPECT imaging (27). The basis for the use of the fragment ubiquicidin 29-41 is its ability to be attracted to the negatively charged bacterial cell wall, itself being positively charged. Ubiquicidin is also produced in activated macrophages and in colonic epithelial cells. In an analysis of the diagnostic performance of ${ }^{99 \mathrm{~m}} \mathrm{Tc}$-ubiquicidin in infection imaging from studies published between 2004 and 2010, a pooled sensitivity and specificity of $94.5 \%$ (95\% confidence interval, $91.2 \%-96.8 \%$ ) and $92.7 \%$ (95\% confidence interval, $80 \%-100 \%$ ), respectively, were reported (28).

The zinc-dipicolylamine analog, a positively charged metal complex that is attracted to the negatively charged bacterial cell wall, is another agent that has been studied in animal models of bacterial infection. It was coupled with ${ }^{111}$ In for imaging and with PSVue 794 (Molecular Targeting Technologies) for florescent imaging in one study (29), which found that both forms of zinc-dipicolylamine analog accumulate intensely at the site of Streptococcus pyogenes infection but only mildly at the site of sterile inflammation. The specificity of zinc-dipicolylamine analog for infection imaging remains a challenge because it is also attracted to the negatively charged membrane of cells undergoing apoptosis or necrosis (30).

Successful treatment of HIV infection reduces the viral load to an undetectable level. Viral replication, however, continues in sanctuary organs. As efforts for HIV cure continue, the need to identify persistence of viral replication in aviremic patients is crucial. A study using immunoPET demonstrated the utility of $7 \mathrm{D} 3-\mathrm{PEG}-{ }^{64} \mathrm{Cu}-\mathrm{DOTA}$, which targets the simian immunodeficiency virus envelop protein gp120 in identifying residual viral replication sanctuary sites of aviremic antiretroviral-treated monkeys (31). This holds great promise for evaluating the effectiveness of therapies directed at HIV cure.

\section{IMAGING MICROBIAL IRON METABOLISM: RADIOLABELED SIDEROPHORES}

Iron is essential for many metabolic processes, including respiration in eukaryotes and many prokaryotes. Siderophores are iron-binding molecules that bacteria and fungi use to scavenge extracellular iron for their metabolism. Several siderophores exist in nature, and many of them have been successfully chelated to radiometals such as ${ }^{67} \mathrm{Ga}$ and ${ }^{111} \mathrm{In}$ (for SPECT imaging) or ${ }^{68} \mathrm{Ga}$ and ${ }^{89} \mathrm{Zr}$ (for PET imaging) (32). ${ }^{67} \mathrm{Ga}$ used to be popular for infection imaging. It has, however, fallen out of favor because of its poor image quality, long imaging period, and high radiation burden to the patients. It has been replaced by ${ }^{68} \mathrm{Ga}$-citrate, a PET tracer with better image quality and favorable dosimetry properties. Vorster et al. recently described its use in imaging infection and inflammation (33). A derivative of deferoxamine was successfully labeled with ${ }^{67} \mathrm{Ga}$ and was shown to localize at the site of Staphylococcus aureus infection (34).

In another study, the use of radiolabeled siderophore in an animal model of invasive fungal infection due to Aspergillus fumigatus was investigated. ${ }^{68} \mathrm{Ga}$ was successfully used to label triacetylfusarinine $\mathrm{C}$ (a siderophore produced by $A$. fumigatus) and ferrioxamine $\mathrm{E}$ (35). Both agents showed good uptake at the site of pulmonary aspergillus infection, with the intensity of uptake correlating with the severity of the infection. No abnormal uptake of either agent was seen in animals without the infection. Identification of an organism-specific siderophore and its labeling with a radiometal holds promise for organism-specific molecular imaging of infection in humans.

\section{IMAGING BACTERIA-SPECIFIC CARBOHYDRATE METABOLISM: ${ }^{18}$ F-FLUORODEOXYSORBITOL}

Enterobacteriaceae are a group of gut-inhabiting gramnegative bacilli capable of causing serious human infection. They are different from other microbial or mammalian cells in their ability to metabolize sorbitol. ${ }^{18} \mathrm{~F}$-fluorodeoxysorbitol has been successfully synthesized from ${ }^{18} \mathrm{~F}-\mathrm{FDG}$, and it showed specific uptake in cultures of Escherichia coli and Klebsiella pneumoniae (36). No uptake of ${ }^{18}$ F-fluorodeoxysorbitol was seen in gram-positive organisms, normal human cells, or cancer cells. Heat-killed Escherichia coli did not accumulate ${ }^{18} \mathrm{~F}$-fluorodeoxysorbitol. The probe was able to differentiate infection due to Enterobacteriaceae from sterile inflammation, and the PET signal disappeared after successful treatment. The safety and biodistribution of ${ }^{18} \mathrm{~F}$-fluorodeoxysorbitol has been demonstrated in a human study (37).

\section{IMAGING MICROBIAL REPLICATION: RADIOLABELED FIALURIDINE}

Fialuridine is a nucleoside analog that is a substrate for the bacterial thymidine kinase enzyme but is not acted on by the human form of the enzyme. This is its basis for use as a potential molecular probe for infection imaging. The 
initial exciting results obtained from imaging skeletal infection have not been replicated in recent human studies. Peterson et al. could not demonstrate incorporation of ${ }^{14} \mathrm{C}$ fialuridine into Pseudomonas aeruginosa DNA in a liquid scintillation counter because Pseudomonas is one of the bacteria without thymidine kinase (38).

\section{IMAGING MICROBIAL BIOLUMINESCENCE: RADIOLABED FLUOROPHORES}

Optical imaging has been extensively used in animal models of infection. Its application in humans has faced many challenges, including toxicity, scattering and absorbance of light within tissue, and the poor signal-to-noise ratio of fluorophores within the human body (39). Using fluorophores, such as indocyanine green, that fluoresce near the infrared region has been helpful in overcoming tissue absorption and scattering of light, hence making imaging of deep infectious processes possible at the skin surface. The feasibility of multiagents in infection imaging using an antimicrobial peptide conjugated to a fluorophore and a radionuclide has been demonstrated (40). Mills et al. have recently reported on an array of optical probes that have been tested in infection imaging (39).

\section{PERSPECTIVE}

Metabolic imaging of infection holds great promise. The focus of its application is shifting from mere diagnosis of infection to prognostication, where response to treatment can be predicted, resistant strains identified, response assessed, and at-risk patients identified and targeted for prevention. The introduction of CT for complementary morphologic imaging has improved the diagnostic performance of metabolic imaging for infection. It is hoped that when PET/MRI and SPECT/MRI achieve greater clinical utility, these hybrid systems may have even more applications in infection imaging. It is also hoped that hybrid molecular probes for multimodality imaging soon may gain clinical relevance for infection imaging. Focused research is pointing toward a time when molecular probes will be able to not only detect infection but also identify the offending organism and its biologic characteristics.

\section{DISCLOSURE}

No potential conflict of interest relevant to this article was reported.

\section{ACKNOWLEDGMENT}

We thank the Department of Nuclear Medicine at the University of Pretoria and NECSA.

\section{REFERENCES}

1. Jain SK. The promise of molecular imaging in the study and treatment of infectious diseases. Mol Imaging Biol. 2017;19:341-347.
2. Govaert GA, IJpma FF, McNally M, McNally E, Reininga IH, Glaudemans AW. Accuracy of diagnostic imaging modalities for peripheral post-traumatic osteomyelitis: a systematic review of recent literature. Eur J Nucl Med Mol Imaging. 2017;44:1393-1407.

3. Kim HO, Na SJ, Oh SJ, et al. Usefulness of adding SPECT/CT to ${ }^{99 \mathrm{~m} T c-}$ hexamethylpropylene amine oxime (HMPAO)-labeled leukocyte imaging for diagnosing prosthetic joint infections. J Comput Assist Tomogr. 2014;38:313319.

4. Muñoz P, Kestler M, De Alacron A, et al. Current epidemiology and outcome of infective endocarditis: a multicenter, prospective, cohort study. Medicine (Baltimore). 2015;94:e1816.

5. Rouzet F, Chequer R, Benali K, et al. Respective performance of ${ }^{18} \mathrm{~F}-\mathrm{FDG}$ PET and radiolabeled leukocyte scintigraphy for the diagnosis of prosthetic valve endocarditis. J Nucl Med. 2014;55:1980-1985.

6. Habib G, Lancellotti P, Antunes MJ, et al. 2015 ESC guidelines for the management of infective endocarditis. Eur Heart J. 2015;36:3075-3128.

7. Palestro CJ. Radionuclide imaging of musculoskeletal infection: a review. J Nucl Med. 2016;57:1406-1412.

8. Miñana E, Roldán M, Chivato T, Martínez T, Fuente T. Quantification of the chromosomal radiation damage induced by labelling of leukocytes with $\left[{ }^{18}\right.$ F]FDG. Nucl Med Biol. 2015;42:720-723.

9. Smids C, Kouijzer IJ, Vos FJ, et al. A comparison of the diagnostic value of MRI and ${ }^{18}$ F-FDG-PET/CT in suspected spondylodiscitis. Eur Infection. 2017;45:4149.

10. Yang H, Zhuang H, Rubello D, Alavi A. Mild-to-moderate hyperglycemia will not decrease the sensitivity of ${ }^{18} \mathrm{~F}$-FDG PET imaging in the detection of pedal osteomyelitis in diabetic patients. Nucl Med Commun. 2016;37:259-262.

11. Lawal I, Sathekge M. F-18 FDG PET/CT imaging of cardiac and vascular inflammation and infection. Br Med Bull. 2016;120:55-74.

12. Ahmed FZ, James J, Cunnington C, et al. Early diagnosis of cardiac implantable electronic device generator pocket infection using ${ }^{18} \mathrm{~F}$-FDG-PET/CT. Eur Heart J Cardiovasc Imaging. 2015;16:521-530.

13. Granados U, Fuster D, Pericas JM, et al. Diagnostic accuracy of ${ }^{18}$ F-FDG PET/ $\mathrm{CT}$ in infective endocarditis and implantable cardiac electronic device infection: a cross-sectional study. J Nucl Med. 2016;57:1726-1732.

14. Sah B-R, Husmann L, Mayer D, et al. Diagnostic performance of ${ }^{18}$ F-FDG-PET/ CT in vascular graft infections. Eur J Vasc Endovasc Surg. 2015;49:455-464.

15. Kagna O, Kurash M, Ghanem-Zouabi N, Keidar Z, Israel O. Does antibiotic treatment affect the diagnostic accuracy of FDG PET/CT studies in patients with suspected infectious processes? J Nucl Med. May 4, 2017 [Epub ahead of print].

16. Keidar Z, Pirmisashvili N, Leiderman N, Nitecki S, Israel O. ${ }^{18}$ F-FDG uptake in noninfected prosthetic vascular grafts: incidence, patterns, and changes over time. J Nucl Med. 2014;55:392-395.

17. Pereira AM, Husmann L, Sah BR, Battegay E, Franzen D. Determinants of diagnostic performance of ${ }^{18} \mathrm{~F}$-FDG PET/CT in patients with fever of unknown origin. Nucl Med Commun. 2016;37:57-65.

18. Bharucha T, Rutherford A, Skeoch S, et al. Diagnostic yield of FDG-PET/CT in fever of unknown origin: a systematic review, meta-analysis, and Delphi exercise. Clin Radiol. 2017;72:764-771.

19. Ankrah AO, van der Werf TS, de Vries EFJ, Dierckx RAJO, Sathekge MM, Glaudemans AWJM. PET/CT imaging of Mycobacterium tuberculosis infection. Clin Transl Imaging. 2016;4:131-144.

20. Stelzmueller I, Huber H, Wunn R, et al. ${ }^{18} \mathrm{~F}-\mathrm{FDG}$ PET/CT in the initial assessment and for follow-up in patients with tuberculosis. Clin Nucl Med. 2016;41:e187-e194.

21. Mattila JT, Beaino W, Maiello P, et al. Positron emission tomography imaging of macaques with tuberculosis identifies temporal changes in granuloma glucose metabolism and integrin $\alpha 4 \beta 1$-expressing immune cells. J Immunol. 2017;199: 806-815.

22. Lefebvre N, Argemi X, Meyer N, et al. Clinical usefulness of ${ }^{18}$ F-FDG PET/CT for initial staging and assessment of treatment efficacy in patients with lymph node tuberculosis. Nucl Med Biol. 2017;50:17-24.

23. Malherbe ST, Shenai S, Ronacher K, et al. Persisting positron emission tomography lesion activity and Mycobacterium tuberculosis mRNA after tuberculosis cure. Nat Med. 2016;22:1094-1100.

24. Esmail H, Lai RP, Lesosky M, et al. Characterization of progressive HIVassociated tuberculosis using 2-deoxy-2-[ $\left.{ }^{18} \mathrm{~F}\right]$ fluoro-D-glucose positron emission and computed tomography. Nat Med. 2016;22:1090-1093.

25. Auletta S, Galli F, Lauri, Martinelli D, Santino I, Signore A. Imaging bacteria with radiolabeled quinolones, cephalosporins and siderophores for imaging infection: a systematic review. Clin Transl Imaging. 2016;4:229-252.

26. Zhang Z, Ordonez AA, Smith-Jones $P$, et al. The biodistribution of $5-\left[{ }^{18} \mathrm{~F}\right]$ fluoropyrazinamide in Mycobacterium tuberculosis-infected mice determined by positron emission tomography. PLoS One. 2017;12:e0170871. 
27. Ebenhan T, Zeevaart JR, Venter JD, et al. Preclinical evaluation of ${ }^{68} \mathrm{Ga}$-labeled 1,47-triazacyclononane-1,4,7-triacetic acid-ubiquicidin as a radioligand for PET infection imaging. J Nucl Med. 2014;55:308-314.

28. Ostovar A, Assadi M, Vahdat K, et al. A pooled analysis of diagnostic value of ${ }^{99 \mathrm{~m}} \mathrm{Tc}$-ubiquicidin (UBI) scintigraphy in detection of an infectious process. Clin Nucl Med. 2013;38:413-416.

29. Liu X, Cheng D, Gray BD, et al. Radiolabeled Zn-DPA as a potential infection imaging agent. Nucl Med Biol. 2012;39:709-714.

30. Bunschoten A, Welling MM, Termaat MF, Sathekge M, van Leeuwen FWB. Development and prospects of dedicated tracers for the molecular imaging of bacterial infections. Bioconjug Chem. 2013;24:1971-1989.

31. Santangelo PJ, Rogers KA, Zuria C, et al. Whole-body immunoPET reveals active SIV dynamics in viremic and antiretroviral therapy-treated macaques. Nat Methods. 2015;12:427-432.

32. Petrik M, Zhai C, Haas H, Decristoforo C. Siderophores for molecular imaging applications. Clin Transl Imaging. 2017;5:15-27.

33. Vorster M, Maes A, van de Wiele C, Sathekge M. Gallium-68 PET: a powerful generator-based alternative to infection and inflammation imaging. Semin Nucl Med. 2016;46:436-447.
34. Ioppolo JA, Caldwell D, Beiraghi O, et al. ${ }^{67} \mathrm{Ga}$-labeled deferoxamine derivatives for imaging bacterial infection: preparation and screening of functionalized siderophore complexes. Nucl Med Biol. 2017;52:32-41.

35. Petrik M, Franssen GM, Haas H, et al. Preclinical evaluation of two ${ }^{68} \mathrm{Ga}-$ siderophores as potential radiopharmaceuticals for Aspergillus fumigatus infection imaging. Eur J Nucl Med Mol Imaging. 2012;39:1175-1183.

36. Weinstein EA, Ordonez AA, DeMarco VP, et al. Imaging Enterobacteriaceae infection in vivo with ${ }^{18} \mathrm{~F}$-fluorodeoxysorbitol positron emission tomography. Sci Transl Med. 2014;6:259ra146.

37. Yao S, Xing H, Zhu W, et al. Infection imaging with ${ }^{18}$ F-FDS and first-in-human evaluation. Nucl Med Biol. 2016;43:206-214.

38. Peterson KL, Reid WC, Freeman AF, et al. The use of ${ }^{14} \mathrm{C}-\mathrm{FIAU}$ to predict bacterial thymidine kinase presence: implications for radiolabeled FIAU bacterial imaging. Nucl Med Biol. 2013;40:638-642.

39. Mills B, Bradley M, Dhaliwal K. Optical imaging of bacterial infections. Clin Transl Imaging. 2016;4:163-174.

40. Welling MM, Bunschoten A, Kuil J, et al. Development of a hybrid tracer for SPECT and optical imaging in bacterial infections. Bioconjug Chem. 2015;26: 839-849. 